\title{
MITC: An Intention-Based Model for Cooperative Resolution of Traffic Conflicts
}

\author{
Alejandro Triana Castañeda \\ Computer Science and System Engineering \\ Pontifical Javeriana University \\ Bogotá, Colombia \\ f.triana@javeriana.edu.co
}

\author{
Enrique González Guerrero \\ Computer Science and System Engineering \\ Pontifical Javeriana University \\ Bogotá, Colombia \\ egonzal@javeriana.edu.co
}

\begin{abstract}
Urban traffic problems have become a quotidian problem that affects many cities in the world. This problem, caused by the exponential increase of vehicles, leads to the appearance of different complications such as environmental pollution, accidents and slow mobility. This work formulates MITC, a model of cooperation focused to conflict resolution for the traffic agents, considering explicit communication of their intentions, allowing them to adjust their decisions intelligently, so as to reduce the conflicts and mitigate traffic congestion.
\end{abstract}

Keywords-Intelligent Traffic Systems; Conflict Resolution; Game Theory; Multiagent Systems

\section{INTRODUCTION}

U RBAN traffic problems have become an everyday problem that affects many cities in the world. The total amount of vehicles in the world is calculated to be about 600 million, with an annual increase of 50 million [18]. Different factors such as the inefficiency in the infrastructure and its planning or a weak public awareness of traffic have increased the complexity of the problem [18]. Traffic problems can be divided into three kinds [10][6]: 1) Mobility issues, related to traveling time, 2) Safety issues, specially focused in preventing accidents, and 3) Environmental issues, generally caused by $\mathrm{CO} 2$ emissions.

Intelligent Transportation Systems (ITS) have emerged as an answer to traffic problems becoming one of the most interesting and promising alternatives within the scientific community [4][5][6]. The ITS aim to apply different artificial intelligence techniques such as Fuzzy Logic [3][7], Neuronal Network [15][16], Evolutionary Computation [14] and, in a more general way, the Agent and Multiagent System paradigm [9][12]. The works on ITS based on Multiagent Systems have covered a great quantity of fronts, among which these can be found: road traffic [7][13], urban traffic control (UTC) [1][2] [4][6][8], and decision support systems [7][17]. In all these solutions, the agents make decisions in an intelligent and cooperative way based in their knowledge of their surroundings.

This paper describes the Intentional Model for Cooperative Traffic (MITC for its name in Spanish). This solution is a traffic model based on Multiagent Systems in which agents cooperate explicitly communicating their intentions in order to solve traffic conflicts. The communication of intentions allows agents to adjust their decisions in an intelligent way to reduce the conflicts generated by the scarcity of resources (highway network) and non-compatible goals (antagonism between vehicles). The conflict resolution is inspired in the benevolence concept, namely the traffic agents with best traffic culture are prioritized. The second section introduces the agent's model and the proposed interaction mechanisms between them. The third section exposes the cooperative model, specifically the conflict resolution protocol. The four section describes the decision making system game theory based, which aims to reduce the traffic conflicts. The experiments that were carried out to evaluate this model are detailed in the last section. Finally, the conclusions are exposed from the perspective of reduction of conflicts between the traffic agents.

\section{Traffic Multiagent System}

This section describes the architecture of the Multiagent System. Initially, the design precepts are presented to describe the general characteristics of architecture. Follow, the characterization of the agents is defined in terms of their main goal. The final part of this section characterizes the agents' interactions and the existing means of communication.

\section{A. Multiagent System's Basic Characteristics}

Urban Traffic systems are highly complex, inherently distributed and have to deal with limited infrastructural resources. Due to these restrictions, the proposed Multiagent model exhibits the following characteristics:

1. Focused in Congestion Problems: its components and its relations aim to lower the conflicts among the traffic agents.

2. Highly Concurrent: it supports the great number of interactions among the agents, which are usually simultaneous.

3. Robust: it controls the handling of exceptional situations such as the damaging of sensors and traffic lights, among others.

4. Scalable: it allows the deployment in cities of different size and complexity.

\section{B. Agents}

This work proposes a model with five agents is describing in the Table 1. Each of the agents of the system are characterized in terms of their main goal, namely their principal function 
inside the system. Likewise, each agent has an alias for quickly reference in the document.

TABLE I. SySTEM AGENTS

\begin{tabular}{|l|l|}
\hline \multicolumn{1}{|c|}{ Name } & \multicolumn{1}{|c|}{ Main Goal } \\
\hline $\begin{array}{l}\text { Traffic Intersection Agent }- \\
\text { TIA }\end{array}$ & $\begin{array}{l}\text { Controls vehicles in crossing } \\
\text { intersections; for instance traffic lights. }\end{array}$ \\
\hline Traffic Sensor Agent - TSA & $\begin{array}{l}\text { Provides traffic information and } \\
\text { generates of metrics of vehicle flow } \\
\text { performance in a vehicle segment. The } \\
\text { vehicle segment refers to the structure } \\
\text { proposed in a Linear Based System } \\
\text { (LBS). }\end{array}$ \\
\hline Driver Control Agent - DA & $\begin{array}{l}\text { Controls the motion of a vehicle going } \\
\text { from a origin point to a destination } \\
\text { point in the shortest possible time. }\end{array}$ \\
\hline $\begin{array}{l}\text { Traffic Area Monitor Agent } \\
\text {-TAMA }\end{array}$ & $\begin{array}{l}\text { Delivers information concerning a } \\
\text { determinate on very large traffic area. }\end{array}$ \\
\hline $\begin{array}{l}\text { Traffic Supervisor Agent }- \\
\text { TSUA }\end{array}$ & $\begin{array}{l}\text { Supervision, support and control of the } \\
\text { decisions of human controllers. }\end{array}$ \\
\hline
\end{tabular}

Accordingly, Fig. 1 illustrates the agent interactions in the proposed model. These interactions involve the existence of last generation technologies such as the detection of pedestrian flow, the presence of sensors (such as GPS), among others. However, some of the mentioned technologies are optional (for example the sensor for pedestrian flow), if available it allows a higher efficiency for the proposed model.

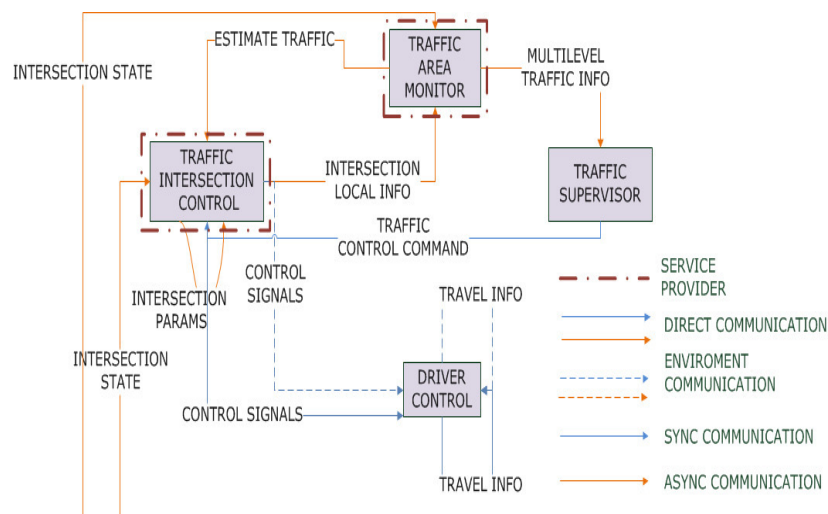

Fig. 1 Agent Interaction

Agent interactions can have different communication mechanisms, as follows:

1. Service Provider: It refers to an agent that provides an specific type of information to different agents. This information can be supplied in two ways:

- Agents can subscribe to the Service Provider and receive the information asynchronously (Async Communication), whenever it is available.

- Agents carry out demands to obtain information synchronously (Sync Communication).

1. Direct Communication: direct and explicit communication, usually through mechanisms like flashing headlights or other non-conventional ones, such as wireless networks.

2. Environmental Communication: Indirect communication across the environment. For instance, the horn or the turn signal lever.

\section{CONFLICT RESOLUTION}

In this section, the protocol of the MITC conflict resolution mechanism is described. Firstly, the concept and the categorization of the intentions that are used in this model are defined. Secondly, the intentions for each of the traffic agents are described. Finally, the characterizations of the traffic conflicts together with the proposed resolution protocol are introduced.

\section{A. Definition of Intentions}

This paper defines an intention as the goals that a traffic agent can have. These goals can follow a hierarchal and recursive classification, as follows:

1. Global Purpose Intentions: corresponds to the global aim of the agent.

2. Deliberative Intentions: refers to those intentions that are subject to the sequence of actions included in the plan of the agent. For example, the driver, according to his knowledge of traffic and exogenous information (news, weather forecast etc.), selects a path that includes several routes to go from his point of origin to his destination point.

3. Immediate Intentions: real-time actions carried out according to nearby traffic conditions. These intentions are motivated by the environment, the agent manage them in a reactive fashion; for instance, a traffic accident or a blockage due to adverse weather conditions.

Accordingly, consider a Multiagent System with $N$ agents and

$1 \leq i \leq N$

- For every agent $a_{i}$ one Global Intention $G_{i}$ exists.

- $G_{i}$ is achieved by a sequence of Deliberative Intentions included in a Plan $P i=\left(P_{i 1}, P_{i 2}, \cdots P_{i P}\right)$ of size $p$ and $1 \leq p \leq P$.

- $P_{i p}$ is carried out by a sequence of $T$ Immediate Intentions $\left(I_{i 1}, I_{i 2}, \quad \ldots I t_{i T}\right)$ and $1 \leq t \leq T$.

In this sense, the previous definition for the intentions can be to apply for the traffic agents' model as shown in TABLE 
II. These definitions let classify the traffic agents like: 1.) Expressive: agents can communicate the intentions for conflict resolution (TIA y DA are expressive agents) and 2.) Support Agents: agents provide information to use in conflict resolution.

TABLE I. TRAFFIC INTENTION AGENTS

\begin{tabular}{|c|c|c|c|}
\hline Agent & $\begin{array}{l}\text { Global Purpose } \\
\text { Intentions }\end{array}$ & $\begin{array}{l}\text { Deliberative } \\
\text { Intentions }\end{array}$ & $\begin{array}{l}\text { Immediate } \\
\text { Intentions }\end{array}$ \\
\hline TSA & $\begin{array}{l}\text { Obtain traffic } \\
\text { information and give } \\
\text { rise to measures that } \\
\text { can determine its } \\
\text { performance. }\end{array}$ & & \\
\hline TIA & $\begin{array}{l}\text { Mitigate vehicle time } \\
\text { delay. }\end{array}$ & $\begin{array}{l}\text { Control } \\
\text { parameters } \\
\text { adjustments } \\
\text { according to } \\
\text { historical } \\
\text { acquisition. }\end{array}$ & $\begin{array}{l}\text { High-beam } \\
\text { switch }\end{array}$ \\
\hline \multirow[t]{5}{*}{$\mathrm{DA}$} & \multirow{5}{*}{$\begin{array}{l}\text { Going from point } A \text { to } \\
\text { point } B \text { in the least } \\
\text { possible time. }\end{array}$} & \multirow[t]{5}{*}{$\begin{array}{l}\text { Travel route } \\
\text { selection. }\end{array}$} & $\begin{array}{l}\text { Right or left } \\
\text { turn. }\end{array}$ \\
\hline & & & $\begin{array}{l}\text { Move } \\
\text { forward }\end{array}$ \\
\hline & & & Brake \\
\hline & & & Accelerate \\
\hline & & & $\begin{array}{l}\text { Change of } \\
\text { lane }\end{array}$ \\
\hline TAMA & $\begin{array}{lr}\text { Deliver } & \text { multilevel } \\
\text { information } & \text { of a } \\
\text { determined } & \text { traffic } \\
\text { area. } & \\
\end{array}$ & & \\
\hline TSUA & $\begin{array}{l}\text { Support human } \\
\text { controlers' decisions }\end{array}$ & $\begin{array}{l}\text { Establish } \\
\text { control rule } \\
\text { per period. }\end{array}$ & \\
\hline
\end{tabular}

$B$.

\section{Conflict Resolution Protocol}

As previously mentioned, traffic conflicts are framed within road infrastructure shortages and agents' antagonist goals. Such conflicts happen in a defined geographic area (e.g. an intersection) and have a limited time duration. Accordingly, in order to solve conflicts the MITC model proposes the following:

1. A conflict has a scope $C S$ denominated conflict set. The scope refers to the set of agents that intervene in the conflict, that is $C S=\left(a_{1}, a_{2}, a_{k} \cdots a_{M}\right)$ with $1 \leq k \leq M \cdot$

2. Every agent $a_{k}$ has a credit $c_{k}$. The credit represents the accumulated benefit that an agent has received when a conflict is solved to his favor.

3. There is an agent initiator of the $a_{i}$ conflict protocol (an agent initiator is any agent traffic that can express its intentions), who communicates an immediate intention $I_{i t}$ of the set of available immediate intentions, that is $I_{i t} \in\left(I_{i 1}, I_{i 2}, I_{i 3} \cdots I_{i T}\right)$ with $0<t \leq T$ and $a_{i} \in C S$.

4. For every agent $a_{i}$, a possibility function $f_{\text {pos }}\left(I_{i t}\right)$ exists, which, given the $I_{i t}$ intention, evaluates the possibility of causing a conflict. The $f_{p o s}$ function complies with the following characteristics:

- It is defined within the range [0,1]. Values close to 1 present a higher possibility of the intention causing a conflict.

- If the value of the function $f_{\text {pos }}$ exceeds a predefined threshold, the dialogue to prevent the conflict is initiated.

5. A CS conflict set has an $a_{m}$ mediator agent associated to it, where $a_{m} \in C S$. The mediator agent is a virtual agent that emerges for to arbitrate the conflict resolution.

6. Each one of the $a_{k}$ agents included in the conflict set generates an bid value $b_{k}$, calculated by a function $f_{b i d}$ such that $f_{b i d}\left(I_{k t}\right)=b_{k}$.

7. For each $a_{k}$ agent there is an associated unit value $u_{k}$ obtained as the result in the conflict resolution process.

8. Every conflict has a unique identifier $t_{m}$. Every message that belongs to the conflict resolution dialogue has to include the identifier associated to it.

Taking into account these definitions and conditions, the proposed conflict resolution protocol is presented in the Fig. 2 and its formulation include the following steps or phases:

1. If the possibility function $f_{\text {pos }}\left(I_{i t}\right)$ of an agent $a_{i}$ exceeds the $U$ threshold, it creates a mediating agent $a_{m}$. The initiating agent sends a conflict init message to the mediating agent attaching the intention $I_{i t}$, the offer $b_{i}$, the accumulated credit $c_{i}$, and the identifiers of the agents within the conflict set CS. 
2. The mediating agent $a_{m}$ forwards the message conflict init to all of the concerned agents $a_{k}$ (with $k \neq i$ and $k \neq m$ ) within the conflict set CS.

3. The agents $a_{k}$ receive the request of a dialogue initiation for conflict resolution and answer with a message conflict response, including their accumulated credit $c_{k}$, and their bid $b_{k}$. Notice that some agents may not respond to the petition of Conflict Resolution because of errors inherent to the communication channel.

4. The mediating agent $a_{m}$ calculates the utility $u_{k}$ for the agent $a_{i}$ and for each agent $a_{k}$. Likewise, $a_{m}$ sends the message conflict result announcing the utility $u_{i}$ to agent $a_{i}$ and the utility $u_{k}$ for each agent $a_{k}$.

5. Finally, the initiating agent sends the message conflict ACK confirming the implementation of the intention.

In this sense, due to the fact that an agent can be involved in different conflicts simultaneously, the following considerations concerning the concurrency issues must be taken into account:

- When an agent $a_{i}$ initiates a conflict dialogue, or an agent $a_{k}$ receives a resolution request, he blocks his availability to participate in any other conflict resolution dialogue. This guarantees that an agent can only participate in one dialogue of conflict resolution at the same time.

- The participation of an agent $a_{i}$ in a conflict is temporary and delimited in time. When time expires, the agent activates his availability in order to participate in any other resolution dialogue.

- $\quad$ For every agent $a_{i}, a_{k} \in C S$ two queues of handling messages exist:

- One queue of incoming messages Qin, which stores the initial resolution messages.' Each message received by the agent is stored in the queue using $t_{m}$ as an identifier.

- One queue to handle the events of a respective conflict Qman. This queue handles the messages for only one conflict simultaneously.
- When the conflict dialogue ends, the agents activate their availability to participate in any other resolution dialogue.

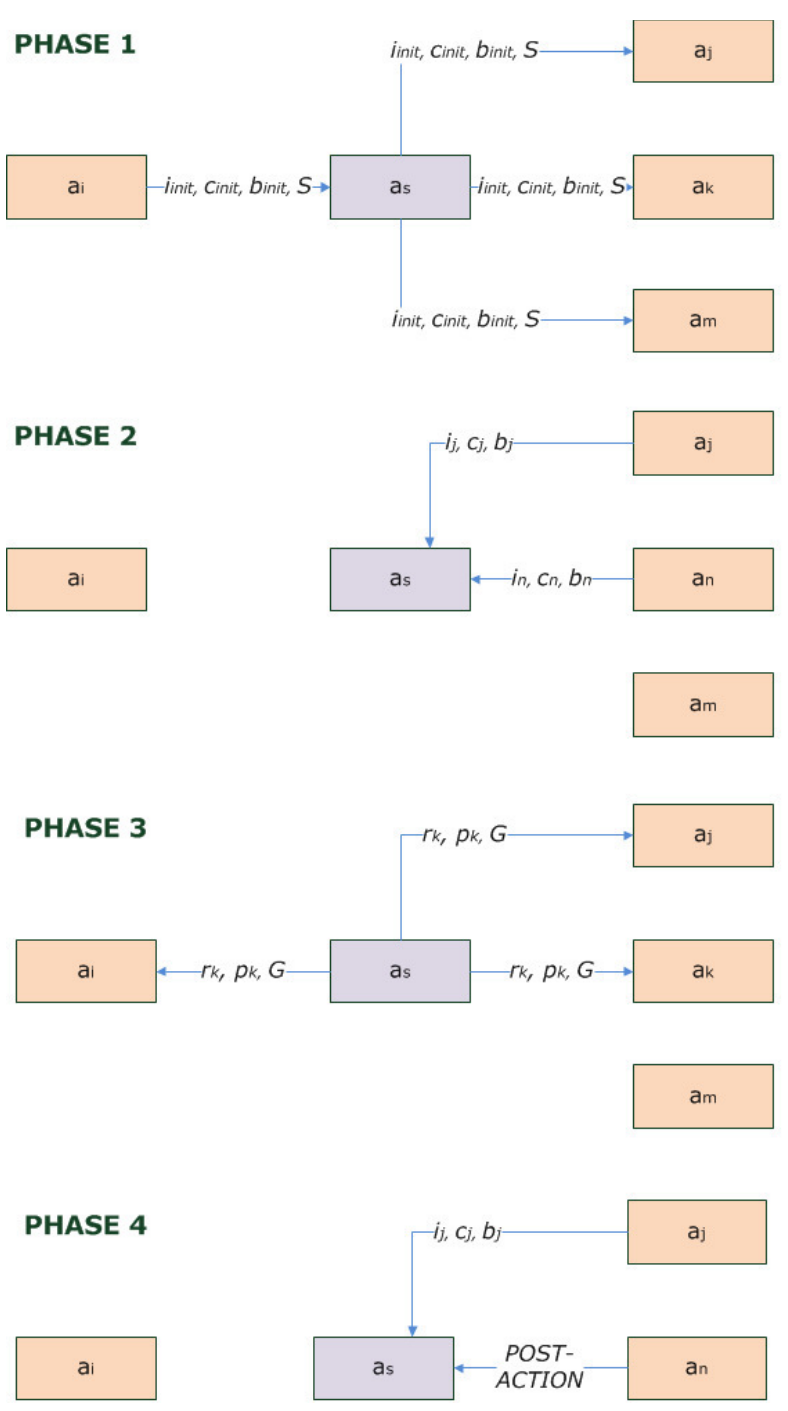

Fig. 2 Resolution Protocol Steps

\section{Decision Model by Game Theory}

This section describes the model of decision making for traffic agents. First, the traffic conflicts are characterized as a game model. Then, the formalization of the game for a traffic conflict is carried out. Finally, the steps for the solution of the conflict are described in terms of the approach of a game in a normal-form.

\section{A. General Assumptions for Traffic Conflict as a Non- Cooperative Model of Game Theory}

MITC proposes a model based on Game Theory to find utility values $u_{k}$ for agents $a_{k}$ that are involved in a conflict set CS such that $C S=\left(a_{1}, a_{2}, a_{k} \cdots a_{M}\right)$ and $1 \leq k \leq M$. 
Traffic conflicts can be described as a model of Game Theory according to the following considerations:

1. These are games of both complete information (the players know completely the strategy of the others, since they communicate their intentions) and perfect information (there is no uncertainty regarding the decisions of the agents).

2. These are games of simultaneous interaction. In other words, each conflict is independent of previous events that happen among the agents.

3. Players: every traffic agent $a_{k} \in C S$.

4. Actions: the vector $I C_{k}=\left(I_{k t},-I_{k t}\right)_{i}$ corresponds to one agent $a_{k}$ where $I_{k t}$ corresponds to the agent's immediate intention and $-I_{k 5}$ corresponds to the non-carrying out of such intention. Be noted that this chapter refers to the terms of action and immediate intention indistinctively.

5. Utility Value: corresponds to the utility value $u_{k}$ obtained by agent $a_{k}$.

\section{B. Traffic Conflict as a Normal-form Game}

One traffic conflict can be characterized as a Normal-form Game, as a tuple $(C S, I C, u)$ where:

1. CS (conflict set) is the finite set of agents $a_{k}$ that take part in the game.

2. IC $=\left(I C_{1}, I C_{2}, I C_{3} \cdots I C_{M}\right)$ is a vector such that $I C_{k t}=\left(I_{k t},-I_{k t}\right)$, wit $h \quad O<k \leq M$.

The intentions $I C_{k t}$ correspond to the set of available actions for agent $a_{k}$ and is denominated action profile for agent $a_{k}$.

3. $u=u_{1}, u_{2} \ldots \ldots \ldots . . u_{M}$, where the utility $u_{k}$ is defined in terms of accumulated credit $c_{k}$ and bid value $b_{k}$.

A more intuitive way to represent a game in a normalform is the bimatrix mechanism. In the bimatrix, the cells contain the utility of each agent for the possible combinations of strategies. Each cell contains two numbers (and therefore the origin of its name), which represent the utility of the agents in such strategy. The figure 3 illustrates the bimatrix for a conflict of change of lanes between two vehicle agents (DA - Driver Agents) $a_{1}$ and $a_{2}$.

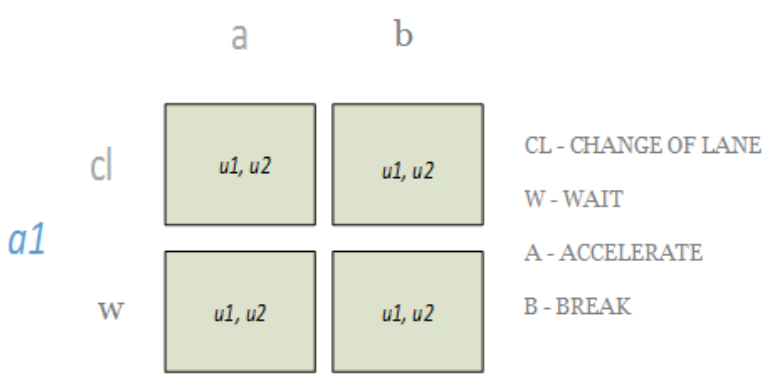

Fig. 3 Bimatrix of a Traffic Game

In general terms, for a traffic conflict with $M$ agents, the actions $I_{1 t}^{*}, I_{2 t}^{*} \cdots I_{M t}^{*}$ form a Nash Equilibrium if for each agent $a_{k}$, the immediate action $I_{k t}^{*}$ is the best action that can be taken by agent $a_{k}$ for the actions of the other $k-1$ players $I_{1 t}^{*}, I_{2 t}^{*}, I_{(k-1) t}^{*}, I_{(k+1) t}^{*} \cdots I_{M t}^{*}$. That is:

$$
\begin{array}{r}
u\left(I_{1 t}^{*}, I_{2 t}^{*}, I_{(k-1) t}^{*}, I_{k t}^{*}, I_{(k+1) t}^{*} \cdots I_{M t}^{*}\right) \\
>u\left(I_{1 t}^{*}, I_{2 t}^{*}, I_{(k-1) t}^{*}, I_{k t}^{\prime} I_{(k+1) t}^{*} \cdots I_{M t}^{*}\right)
\end{array}
$$

For each possible action $I_{k t}$ in $I C_{k t}, I_{k t}$ is a solution for:

$$
\max \left(I_{1 t}^{*}, I_{2 t}^{*}, I_{(k-1) t}^{*}, I_{k t}^{*}, I_{(k+1) t}^{*} \cdots I_{M t}^{*}\right)
$$

\section{Strategy of Nash Equilibrium Calculation}

During steps 3 and 4 of the Conflict Resolution Protocol the mediating agent receives the offers of the agents in the conflict set and must solve the conflict calculating the corresponding Nash equilibrium. The strategy for this calculation given an immediate intention, consists of the following steps:

1. The bids $b_{1}, b_{2}, b_{3}, b_{4} \cdots b_{M}$ are obtained. The bid function $f_{b i d}\left(I_{k t}\right)=b_{k}$ for an agent $a_{k}$ follows these criteria:

a. Each intention $I_{k t}$ has one base bid value associated $w_{k t}$ with $w_{k t} \in Z$. The base bid value can be seen as the importance of the intention within the system's context (e.g. an ambulance can have a greater importance in its intentions than private cars).

b. Each agent $a_{k}$ has a benevolence coefficient $v_{k}$ with $v_{k} \in Z$. For more 
information about the benevolence calculation see section 5.3.

c. Each bid is attenuated by the accumulated credit $c_{k}$. The higher the accumulated credit value, the lesser the bid value $b_{k}$ is. This approach allows controlling those agents that intend to abuse of their benevolence to accumulate excessive credit.

Accordingly, the function for the bid is calculated as:

$b_{k}=f_{\text {bid }}\left(I_{k t}\right)=\left(w_{k t} * v_{k}\right)\left(\frac{1}{c_{k}}\right)$ para $c_{k}>0$

2. The mediating agent $a_{m}$ calculates the game bimatrix $T_{i k}$ for every couple of agents $a_{i}$ (initiating agent) and $a_{k}$ with $i \neq k \cdot$ The calculation of the utilities for the bimax is based on the following conditions:

- If the intention $I_{i l}$ is chosen and not $I_{k l}$, then $u_{i}=b_{i}-b_{k}, u_{k}=b_{k}+b_{i}$

- If the intention $I_{k l}$ is chosen and not $I_{i l}$, then $u_{k}=b_{k}-b_{i}, \quad u_{i}=b_{i}+b_{k}$

- If $I_{i l}$ and $I_{k l}$ are chosen, then $u_{i}=-b_{i}, \quad u_{k}=-b_{k}$

- If $I_{i l}$ and $I_{k l}$ are not chosen, then $u_{i}=0, u_{k}=0$

1. For each matrix $T_{i k}$ the corresponding Nash equilibrium is calculated as $E q_{i k}$.
2. All equilibriums $E q_{i k}$ are obtained, where the intention $I_{i t}$ of the initiating agent $a_{i}$ is selected. Afterwards, the equilibrium $E q_{i k}$ is selected as the one that produces the maximum utility $u_{i}$.

3. The agents $a_{i}$ and $a_{k}$ modify their accumulated credit as follows:

$$
c_{i}=c_{i}+u_{i}, \quad c_{k}=c_{k}+u_{k}
$$

The calculation of Nash equilibrium includes certain characteristics to be taken into account:

- There can be situations in which there is no equilibrium, in which case there is no conflict resolution.

- The state for $u_{i}=-b_{i}$ and $u_{k}=-b_{k}$ can never correspond to an equilibrium; namely, the state in which both agents comply with their intention simultaneously is omitted.

\section{RESUlts}

This chapter describes the experiments carried out to validate the proposed MITC model. First the scenario design for the simulation is presented in order to, later on, present the results of the conducted experiments.

\section{A. Simulation Scenario}

The validation experiments use the scenario of Crossing on a Slow Lane, which is described in terms of figure 4 as follows:

- From point 1 to point 2 there is a distance of 1400 meters.

- From point $\mathbf{1}$ to point $\mathbf{3}$ there is a distance of 1408.93 meters.

- The scenario is comprised of six roads R1, R2, R3, R4, R5, y R6.

- $\quad \mathrm{R} 1$ and R5 have a length of 800 meters with two lanes in each one of them. 
- $\quad$ R2 has a length of 200 meters and 5 lanes.

- $\quad$ R4 has a length of 408.1 meters and 1 lane. It has a connection at the final point of R2.

- $\quad$ R3 and R6 have a length of 400 meters.

\section{B. Results}

During the first part of the protocol, 5 of the experiments were carried out using the native behavior included in the simulator and 5 experiments were carried out using the cooperative model with the following characteristics:

- 10 repetitions were carried out for each experiment.

- The flow of vehicles was varied (HIGH, MEDIUM-HIGH, MEDIUM-LOW, LOW) with a duration of the experiment of 3600 seconds and a 0.2 time step for the simulation'.

- The variables Resolution Activation and Benevolence were established in HIGH.

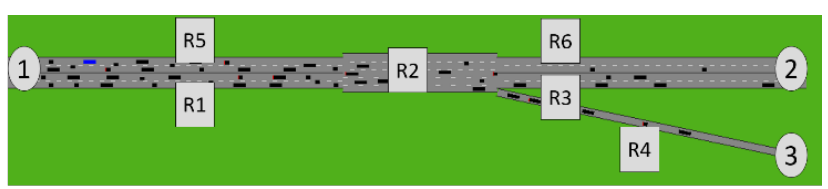

Fig. 4 Simulation Scenario

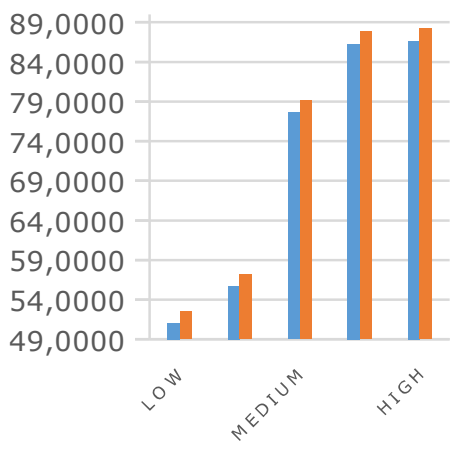

FLOW VEHICLES

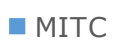

nO

COOPERATION
Fig. 5 Time Comparison

\footnotetext{
${ }^{1}$ The duration constitutes the time that the simulation lasts until it reaches 3600 (it represents one hour in MovSim) with an increasing value by cycle of 0.2 . That is to say, in this case 18000 cycles of simulation would be carried out.
}

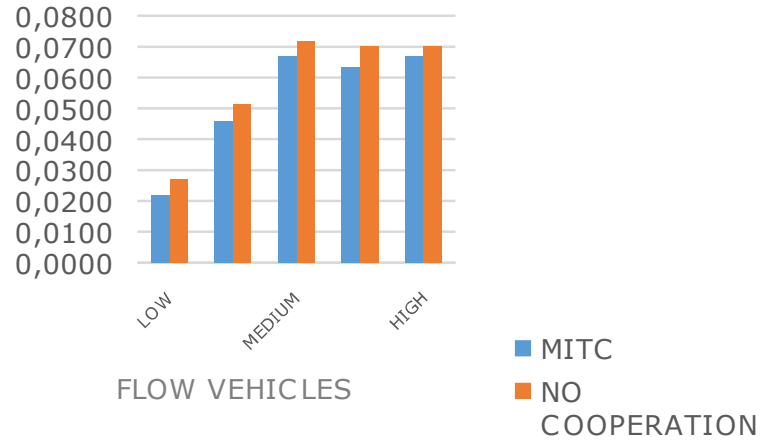

Fig. 6 Consumption Comparison

According to figures 5 and 6 , the MITC model provides an improvement of travel time of $2.17 \%$ per vehicle and of $8.8 \%$ for energy consumption. At first glance these indicators pose an improvement in a local scenario of 1400 meters and, taking it to a scenario with higher dimensions (e.g. a metropolitan area), it may represent great benefits.

The second part of the experiments was focused in the analysis of the Activation Resolution and Benevolence variables. These variables determine the behavior of the model towards exceptional situations, such as infrastructure communication errors and traffic agents with lack of collaborative culture.

As can be observed in Fig. 7 and Fig. 8, the MITC model has a similar behavior in comparison with the results observed in the basic behavior (the simulation without the cooperative model included), when the Activation of the Resolution has values LOW and MEDIUM. This means that the model can cohabit, without negatively affecting the performance of the system, in mixed scenarios that not only include cooperation but also indifference.

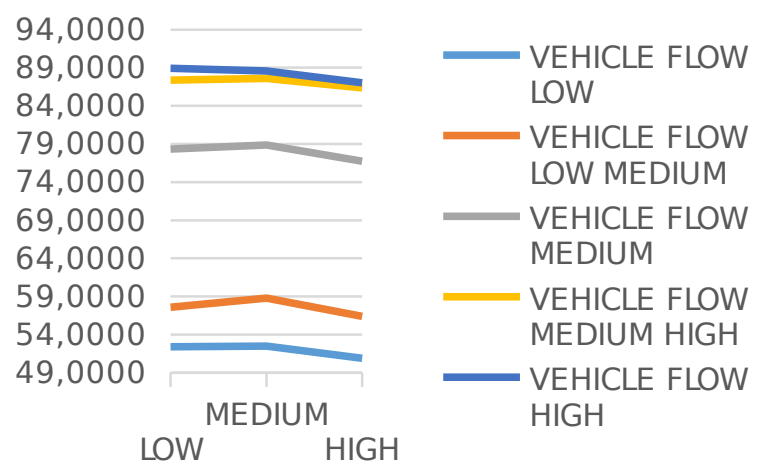

Fig. 7 Results Comparison Activation Resolution - Travel Time 


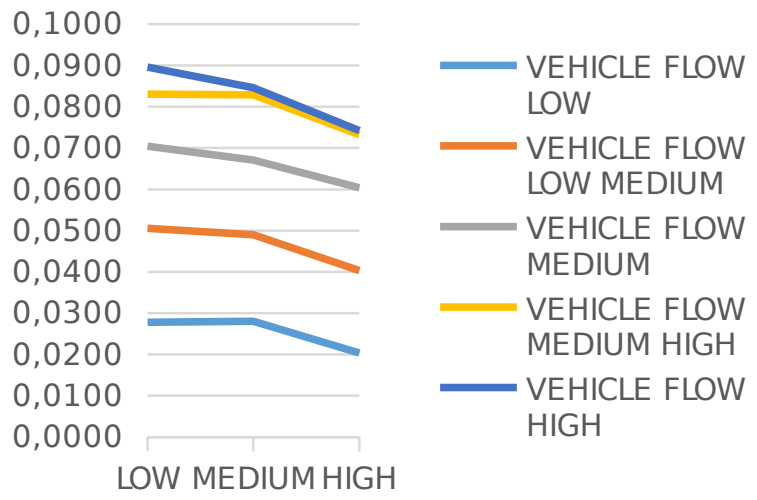

Fig. 8 Results Comparison Activation Resolution - Consumption

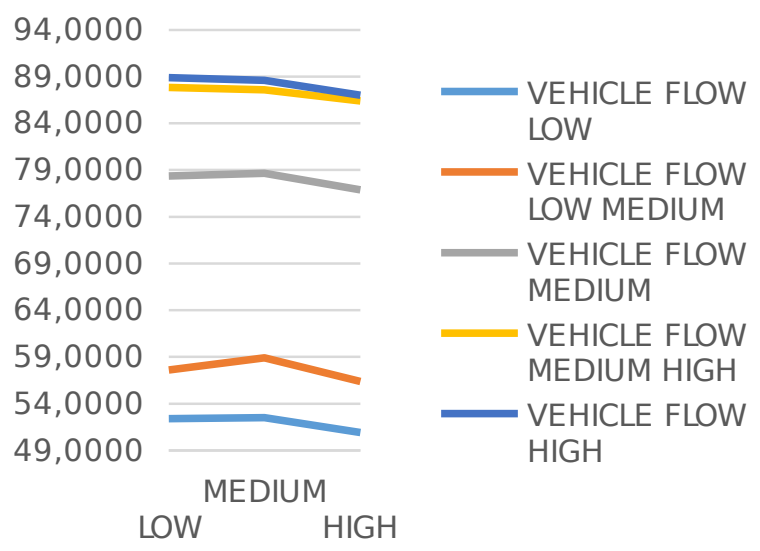

Fig. 9 Comparison Results Benevolence - Travel Time

Likewise, the results for the Benevolence variable can be observed in Fig. 9 and 10. Only when there is higher benevolence (specifically, for HIGH values), traffic conditions improve significantly. This highlights the importance of traffic culture in a city.

\section{CONCLUSIONS}

The design and construction of an Intention-Based Model for Cooperative Resolution of Traffic Conflicts offers a different approach in order to solve congestion problems. MITC allows explicitly expressing the cooperative mechanisms between traffic agents in scenarios where conflicts are presented, according to shared resources and according to conflicting goals. In this sense, MITC provides a set of advantages:

1. The Multiagent Model is designed to tolerate different requirements given by an Urban Traffic System, such as concurrency, scalability and its complexity. In computational terms, MITC allows

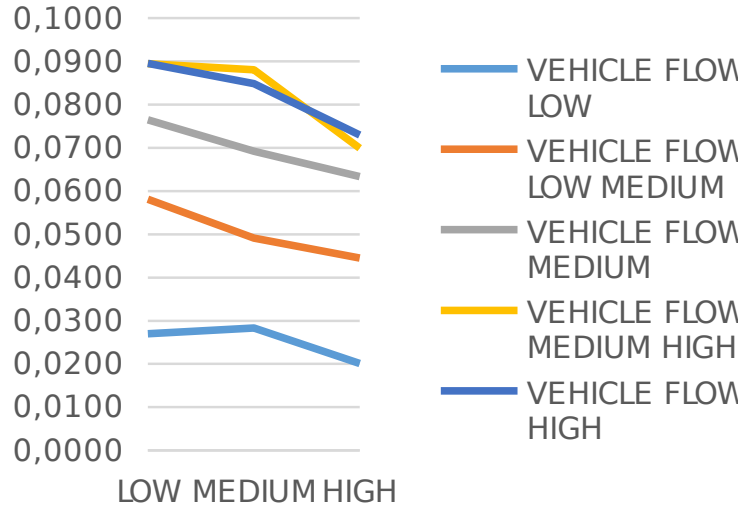

Fig. 10 Comparison Results Benevolence - Consumption

approaching traffic problems in a distributed way, which brings important advantages in terms of availability and fault tolerance.

2. The Multiagent Model poses a Conflict Resolution Protocol that supports the essential characteristics such as concurrency and its temporality.

3. The Multiagent Model covers transversally every aspect of a Traffic System, including control areas, administration and supervision.

4. The definition of the concept of Intention and its three level hierarchies allows modeling the characteristics of traffic agents in a manner that is natural and closer to reality.

5. The Decision Making Model based in Game Theory guarantees the solution of conflict in a rational and balanced way. Additionally, its inspiration in the concept of benevolence allows analyzing essential aspects such as public traffic conscience.

This work opens the door to a great number of applications. For instance, in the development of campaigns for intelligent traffic and traffic culture, it provides formal and measurable elements in terms of travelling time. Likewise, it would be very helpful to include experiments that determine the decrease of accidental rates through MITC on behalf of incorporated elements of road safety that give as a result a pedagogic frame for urban traffic. In this sense and although in Latin American cities traffic networks are far from implementing technologies such as inter-vehicular nets or smart vehicles, MITC is an adaptable model that can be implemented partially. Therefore, designing a System of Urban Traffic Control becomes interesting, posing options for intentional traffic lights and its respective strategies for conflict solving. In a similar way, it is possible to generate a system of traffic recommendations in real time that can 
assist users while they drive their vehicles. Likewise, the model can be extended with a realistic Deliberative Intentions implementation, where the model express a coherent traceability across of intention hierarchy.

MITC is a model with a significant impact when implemented in real traffic scenarios, since it can mitigate some of the factors that have negative effects in the quality of life of people. For example, it can decrease atmospheric pollution, given that vehicles would spend less time on the streets, and it can decrease environmental noise, as it prevents the emission of sound signals of vehicles, as conflicts can be solved automatically.

\section{REFERENCES}

[1] J. L. Adler and V. J. Blue. A cooperative multi-agent transportation management and route guidance system. Transportation Research Part Cemerging Technologies, 10(5-6):433-454, October 2002.

[2] J. L. Adler, G. Satapathy, V. Manikonda, B. Bowles, and V. J. Blue. A multi-agent approach to cooperative traffic management and route guidance. Transportation Research Part B-methodological, 39(4):297-318, May 2005.

[3] T. Akiyama and M. Okushima. Advanced fuzzy traffic controller for urban expressways. International Journal of Innovative Computing Information and Control, 2(2):339-355, April 2006

[4] P. G. Balaji, X. German, and D. Srinivasan. Urban traffic signal control using reinforcement learning agents. Iet Intelligent Transport Systems, 4(3): 177-188, September 2010.

[5] M. Bielli, G. Ambrosino, and M. Boero. Artificial intelligence applications to traffic engineering. Vsp, 1994.

[6] J. C. Burguillo-Rial, P. S. Rodriguez-Hernandez, E. CostaMontenegro, and F. Gil-Castineira. History-based selforganizing traffic lights. Computing and Informatics, 28(2):157-168, 2009.

[7] B. Chen and H. H. Cheng. A review of the applications of agent technology in traffic and transportation systems. Ieee Transactions On Intelligent Transportation Systems, 11(2):485-497, June 2010.
[8] R. S. Chen, D. K. Chen, and S. Y. Lin. Actam: Cooperative multi-agent system architecture for urban traffic signal control. Ieice Transactions On Information and Systems, E88D(1):119-126, January 2005.

[9] M. C. Choy, D. Srinivasan, and R. L. Cheu. Cooperative, hybrid agent architecture for real-time traffic signal control. Ieee Transactions On Systems Man and Cybernetics Part A-systems and Humans, 33(5):597-607, September

2003.

[10] R. Fernandes. A BDI-based approach for assessment of drivers decision-making in commuter. $\mathrm{PhD}$ thesis, Universidade Federal Do Rio Grande Do Sul, nov 2002.

[11] Enrique Gonzales and Cesar Bustacara. Desarrollo de Aplicaciones Basadas en Sistemas Multiagentes. 2007.

[12] J. Z. Hernandez, S. Ossowski, and A. Garcia-Serrano. Multiagent architectures for intelligent traffic management systems. Transportation Research Part C-emerging Technologies, 10(5-6):473-506, October 2002. [13] J. Hillenbrand, A. M. Spieker, and K. Kroschel. A multilevel collision mitigation approach - its situation as sessment, decision making, and performance tradeoffs. Ieee Transactions On Intelligent Transportation Systems, 7(4):528-540, December 2006.

[14] T. Ma and B. Abdulhai. Genetic algorithm-based optimization approach and generic tool for calibrating traffic microscopic simulation parameters. Intelligent Transportation Systems and Vehicle-highway Automation 2002: Highway Operations, Capacity, and Traffic Control, (1800):6-15, 2002.

[15] D. Srinivasan, M. C. Choy, and R. L. Cheu. Neural networks for realtime traffic signal control. Ieee Transactions On Intelligent Transportation Systems, 7(3):261-272, September 2006.

[16] H. B. Yin, S. C. Wong, J. M. Xu, and C. K. Wong. Urban traffic flow prediction using a fuzzy-neural approach rid a-7258-2008. Transportation Research Part C-emerging Technologies, 10(2):85-98, April 2002.

[17] N. Zhang, F. Y. Wang, F. H. Zhu, D. B. Zhao, and S. M. Tang. Dynacas: Computational experiments and decision support for its. Ieee Intelligent Systems, 23(6):19-23, November 2008.

[18] D. Zhao, Y. Dai, and Z. Zhang. Computational intelligence in urban traffic signal control: A survey. Systems, Man, and Cybernetics, Part C: Applications and Reviews, IEEE Transactions on, PP(99):1 -10, 2011 